\title{
Three-dimensional Segmentation of Vessels from Intensity In-homogeneous Medical Images
}

\author{
Faiza Bukenya \\ School of Computer Science \\ University of Nottingham \\ Nottingham, UK \\ Josef Ehling \\ Department of Experimental Molecular Imaging \\ Aachen University \\ Aachen, Germany
}

\author{
Amir Awwad \\ School of Medicine \\ University of Nottingham \\ Nottingham, UK \\ Henryk Faas \\ School of Medicine \\ University of Nottingham \\ Nottingham, UK
}

\author{
Jinming Duan \\ School of Computing \\ Imperial College London \\ London, UK
}

\author{
Li Bai \\ School of Computer Science \\ University of Nottingham \\ Nottingham, UK
}

\begin{abstract}
Blood vessel segmentation helps to know the progress of the disease during diagnosis. The presence of intensity in-homogeneity, conglutination tissue and noise in medical images has led to difficulty in extraction of different sizes of blood vessels, difficulty in separating vessels for further analysis (such as quantification of angiogenesis), difficulty in distinguishing vessels from non vessels. Most of the available techniques are 2D-based. Despite the fact that 2D-based segmentation is easy, it doesn't provide full information about anatomic structure. Most of the available 3D vessel segmentation techniques require human intervention and fail to segment different sizes of vessels. In this paper, a 3D hybrid approach for segmentation has been developed, based on white top hat scale space bilateral hessian vessel enhancement filter and hysteresis threshold method combined with multi-threshold Otsu method. The hybrid method can address noise and intensity in-homogeneity problem, as a result, more vessels of different sizes are detected. The method can also incorporate spatial information, abnormalities in the vessels are detected. Vessels of different sizes are separated to ease further analysis. Conglutination tissue (that obstruct segmentation process) is eliminated to ease extraction of different sizes of vessels.
\end{abstract}

Index Terms - three-dimension, segmentation, medical imaging, blood vessels, image processing

\section{INTRODUCTION}

Blood vessel segmentation is important for diagnosing cardiovascular disease, monitoring treatment response and for treatment planning, including angioplasty and emergency stent placement [1], [2], [3], [4], [5]. In clinical practice, this is based on static $3 \mathrm{D}$ images which allow a more specific analysis of surgical outcome as compared to dynamic images [6]. Dynamic images are used to determine the presence of instability. But manual vessel segmentation is time-consuming, prone to errors and often requires manual user intervention. Therefore, automated methods are indispensable. In previous work, approaches introduced for vessel segmentation employ model based approaches [7], [8], [9], [10], [11], graph based approaches [12], [13], [14], [15], [16], [17], [18], [19], [20], tracking based approaches [21], [22], [23], [24], [25], [26], and level set approach [27], [28], [29], [30], [31], hessian based approaches [32], [33], [34], [35], [23], [36]. However, these approaches are slow, computationally expensive, involve human intervention, face difficulty in distinguishing vessels from non-vessels and segmenting different sizes of vessels due to presence of conglutination tissue [37], intensity inhomogeneity and noise. In addition, some of these approaches have been developed only for 2D images while others that claim to be $3 \mathrm{D}$ based, segment $2 \mathrm{D}$ slices of $3 \mathrm{D}$ image data and later reconstruct $2 \mathrm{D}$ image planes in to $3 \mathrm{D}$ image which lead to inconsistencies in results [7], [38], [39], [40], [41]. Furthermore, some of these approaches segment parts of vessels rather than the whole organ vasculature. Fig. 1, demonstrates the intensity in-homogeneity problem in 3D brain images.
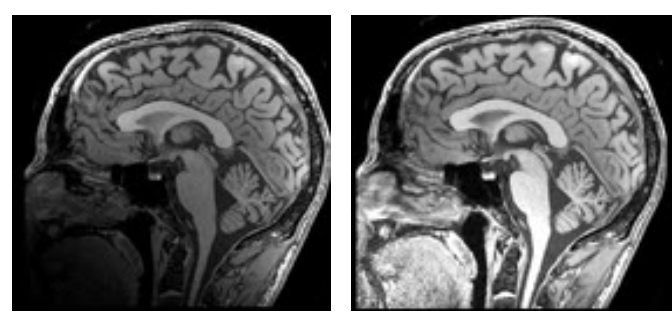

Fig. 1. Illustration of intensity in-homogeneity in MRI brain image planes

In this paper, a hybrid approach has been developed based on white top hat scale space bilateral hessian vessel enhancement filter and combination of hysteresis threshold method and multi-threshold Otsu method for extraction of the full organ vasculature in medical images that suffer from intensity in-homogeneity problem and noise. The major idea of this algorithm is as follows: we have segmented different sizes of vessels using different methods. white top hat scale space bilateral hessian vessel enhancement filter (WTHBHVEF) is used to segment small to medium vessels, multi-threshold Otsu method combined with hysteresis threshold method are used to extract medium to big vessels. Image addition operation is employed to obtain a whole human or animal organ vasculature. This is done to exploit the capabilities of each 
method. For example, multi-threshold Otsu method lead to incorporation of spatial information and also extraction of strong edged big to medium vessels. Hysteresis threshold method is combined with multi-threshold Otsu method during segmentation of big to medium vessels to deal with problem of cutting vessels. As compared to white top-hat scale space hessian vessel enhancement filter (WTHHVEF) in [41], white top hat scale space bilateral hessian vessel enhancement filter (WTHBHVEF) analyze the local behaviour of an image using second order information (Hessian) of white top-hat morphological operation and bilateral filter method rather than only white top-hat morphological operation during extraction of small vessels. This enables us to address intensity inhomogeneity problem, noise as well as maintain size and shape of small vessels. Compared to other segmentation algorithms, this algorithm has given better result. For example, the method is able to deal with intensity in-homogeneity and noise as a result more vessels are detected. The method is capable of eliminating the skull to ease further analysis. The method is able to separate different sizes of vessels to ease further analysis during quantification of angiogenesis. The method incorporates spatial information, abnormalities in vessels are evident. The method guarantees high quantification or classification accuracy, as the method uses bilateral filter which is more accurate than Gaussian filter [42]-[45].

The rest of the paper is structured as follows: Section II discusses the proposed methodology; Section III presents the experimental results; Section IV discusses segmentation results; Section V concludes the paper.

\section{Methods AND MATERIALS}

In order to avoid false detections during segmentation of blood vessels, the images are segmented while considering the fact that we are extracting vessels from three dimensional images with intensity variations.

In our approach, segmentation is carried out two times to ensure extraction of vessels of different sizes. Compared to the method in [41], this method takes advantage of WTHBHVEF during the extraction of small to medium vessels and hysteresis thresholding method with multi-threshold Otsu method during the extraction of big to medium vessels. Image addition operation is performed to generate a whole human or animal organ vasculature.

\section{A. Multi-threshold Otsu Method}

Spatial information is an important aspect as it can improve outbreak detection performance [46]. we chose to utilize 3D multi-threshold Otsu method, which utilizes spatial information during segmentation. By using multi-threshold Otsu method, we are also benefiting from other advantages such as it is fast [47], [41], [48], [49], consumes less storage space [50], [51], ease of implementation [52], [51], [53], [48], [54], [55], [56], [57], [58], [59] and noise resistance [59].

Multi-threshold Otsu method is an adaptive threshold segmentation method that divides the grayscale image in to two classes (namely background and foreground) basing on the image characteristics. The class error is used to show the difference between the classes. The higher the error the more the objects are unlikely to belong to the same class, whilst on the other-hand the lower error the more the objects are likely to belong to a different class [54], [41]. Like other methods, multi-threshold Otsu method has its shortcomings such as the problem of cutting vessels. Compared to the method in [41], we combine hysteresis thresholding method in [60] with multithreshold Otsu method in [54], [41] to deal with the problem of cutting vessels to detect more vessels. Hysteresis thresholding method is also used to get rid of the skull for proper diagnosis and further analysis. We used Multi-threshold Otsu method in [54], [41].

\section{B. Hysteresis thresholding}

In medical image analysis, a method that can segment different sizes of vessels is considered ideal as it helps during diagnosis in cases where angiogenesis is involved to determine the patients response to treatment. We therefore employ hysteresis thresholding to be used with multi-threshold Otsu method to ensure accurate extraction of big to medium vessels. Hysteresis thresholding has consistently been able to outperform absolute thresholding methods because of capability to deal with uncertainties during edge detection. It also detect absence of pixels in true edges even in presence of intensity in-homogeneity [61]-[65]. We use hysteresis thresholding method in [60].

\section{White top-hat scale space bilateral hessian based vessel enhancement filter}

One of the challenges faced by researchers is segmenting and maintaining edges of small vessels especially in presence of intensity homogeneity and noise. we utilize white top-hat scale space hessian based vessel enhancement filter in [41] because of its capability to separate different vessels, enhance edges, correct illumination to uncover the objects rendered absent in the images and also get rid of unwanted tissues in MRI image [41], [66]-[68].

A multiscale approach is employed with the aim of extracting detailed information at various scales. The size of structuring element depends on the scale (scale specified in Frangi hessian based vessel enhancement filter). Increase in the scale leads to increase in the structuring element allowing detailed extraction of information at various scales. In this paper, we have used a disk structuring element [69]. Given Frangi hessian based vessel enhancement filter scale space of levels $\mathrm{n}=1,2, \ldots, \mathrm{L}$. Where a level with index $\mathrm{L}$ corresponds to a white top-hat transform with structuring element size $K_{n}$. $K_{n}$ increases with increase in L. A white top-hat scale space morphological operation $W T H_{n}$ is given by:-

$$
W T H_{n}=I-\left(I \circ K_{n}\right)
$$

Where $I$ is the image, $K_{n}$ denote the structuring element used at level $\mathrm{n}$.

In white top-hat scale space hessian based vessel enhancement filter [41], the local behaviour of an image is analyzed 
using second order information (Hessian) of white top-hat morphological transform operation rather than Gaussian convolution. The eigenvalues obtained from hessian matrix are used to extract tubular structures (vessels). The hessian matrix at point $x$ at scale $n$, is given by:-

$$
H(I, x)=\frac{\partial^{2} I_{n}}{\partial x^{2}}=I(x) \times \frac{\partial^{2} W T H_{n}(x)}{\partial x^{2}}
$$

where $W T H_{n}=I-M(I)$.

Compared to equation(2), Our equation(3) incorporates bilateral filter to build and maintain strong edges of small vessels as well as deal with the noise introduced by top-hat morphological transform operation during segmentation.

$$
H(I, x)=\frac{\partial^{2} I_{n}}{\partial x^{2}}=I(x) \times \frac{\partial^{2} W T H_{n}(x) B_{n}(x)}{\partial x^{2}}
$$

Where $B_{n}$ is bilateral filter in equation (6).

To distinguish tubular structures from blob-like or plate-like structures and background. A dissimilarity measure in equation (5) is used.

$f(x)=\left\{\begin{array}{l}0, \quad \text { if } \lambda_{2}>0 \text { or } \lambda_{3}>0 \\ \left(1-\exp \left(-\frac{R_{a}^{2}}{2 \alpha^{2}}\right)\right) \exp \left(-\frac{R_{b}^{2}}{2 \beta^{2}}\right)\left(1-\exp \left(-\frac{S^{2}}{2 C^{2}}\right)\right)\end{array}\right.$

where

$S=\sqrt{\lambda_{1}^{2}+\lambda_{2}^{2}+\lambda_{3}^{2}}, R_{a}=\left|\frac{\lambda_{2}}{\lambda_{3}}\right|,\left|\frac{\lambda_{1}}{\sqrt{\left|\lambda_{2} \lambda_{3}\right|}}\right|$

The above vesselness measure uses filter responses at different scales taking the maximum response:-

$$
V_{F}(X)=\max _{\delta_{\min } \leq \delta \leq \delta_{\max }} f(x)
$$

\section{Bilateral Filter}

One of the main challenges faced during segmentation is denoising and maintaining edges of small vessels. Some of the available denoising techniques fail to maintain edges of small vessels while denoising, others that succeed in keeping vessels end up changing the size and shape of vessels which in turn lead to poor quantification results, for example recently [42] compared the impact of Gaussian and bileteral filter on quantification results. Both methods were able to denoise images however, the quantification results showed that bilateral filter was able to filter the image without changing size and shape of the object.

Bilateral filtering is a non-local diffusion process that was proposed by Tomasi and Maduchi [70], [45]. Unlike Gaussian filter, Bilateral filter uses two Gaussian filter components to guide diffusion during vessel segmentation [43]-[45]. The Gaussian function of space used in bilateral filter ensures that only pixels in a spatial neighbourhood are considered while Gaussian component employed to address intensity inhomogeneity ensures that only pixels of similar intensities are considered to compute the blurred intensity value to avoid blurriness and also preserves size and shape of edges of vessels [43], [44]. Bilateral filter is also widely used because of its capability to denoise the image even in presence of intensity in-homogeneity [45]. In this paper, we use the bilateral filter in [45]. The bilateral filtering $\mathrm{B}$ of an image $f(i): i \in \mathbb{I}, \mathbb{I}$ being the finite rectangular domain of $\mathbb{Z}^{2}$ is given by [45]:-

$$
f_{B}(i)=\frac{\sum_{j \in \Omega} w(j) g_{\sigma r}(f(i-j)-f(i)) f(i-j)}{\sum_{j \in \Omega} w(j) g_{\sigma r}(f(i-j)-f(i))}
$$

where

$$
g_{\sigma r}(t)=\exp \left(-\frac{t^{2}}{2 \sigma_{r}^{2}}\right)
$$

The spatial filter is a Gaussian:

$$
w(i)=\exp \left(-\frac{\|i\|^{2}}{2 \sigma_{s}^{2}}\right)(i \in \Omega)
$$

The domain $\Omega$ of the spatial kernel is a square neighbourhood, $\Omega=[-W, W] \times[-W, W]$, where $W=3 \sigma_{s}$ for the Gaussian filter. The translated kernel $g_{\sigma r}(t-\tau)$ in equation(10) can be written as

$$
g_{\sigma r}(t-\tau)=\exp \left(-\frac{t^{2}}{2 \sigma_{r}^{2}}\right) \exp \left(-\frac{t^{2}}{2 \sigma_{r}^{2}}\right) \exp \left(\frac{t \tau}{\sigma_{r}^{2}}\right)
$$

where $t=f(i-j)$ and $\tau=f(i)$. For a fixed translation $\tau$, there are three function $t$. The performance of the filter depends on three terms. The first term is a scaling factor, the second term is a Gaussian centered at the origin. the second contributes to the bell shape of the translated Gaussian. The third term is a monotonic exponential that increases or decreases depending on the sign of translation $\tau$. This term helps in translating the Gaussian to $t=\tau$ [45]. Like in other PDE based segmentation techniques (such as anisotropic diffusion), the notion of time evolution of bilateral filter is related to a notion of scale. Like in [45], the Bilateral method used in this paper splits and approximates using Taylor polynomials. The Taylor expansion of the exponential term about the origin is given by:-

$$
\exp \left(\frac{\tau t}{\sigma_{r}^{2}}\right)=\sum_{n=0}^{N-1} \frac{1}{n !}\left(\frac{\tau t}{\sigma_{r}^{2}}\right)^{n}+\text { higher }- \text { orderterms }
$$

The higher-order terms are ignored to obtain the product of a bivariate Gaussian and a polynomial:

$$
\phi_{N, \sigma_{r}}(t, \tau)=\exp \left(-\frac{t^{2}+\tau^{2}}{2 \sigma_{r}^{2}}\right)\left[\sum_{n=0}^{N-1} \frac{1}{n !}\left(\frac{t \tau}{\sigma_{r}^{2}}\right)^{n}\right]
$$

where $\mathrm{N}$ is its approximation order. The point wise convergence is given by:-

$$
\lim _{n \rightarrow \infty} \phi_{N, \sigma_{r}}(t, \tau)=g_{\sigma r}(t-\tau)
$$

\section{EXPERIMENTAL RESULTS}

We tested the proposed method on clinically relevant 3D original medical images. The images used include: 2 contrastenhanced MR angiography(CE-MRA) human neck and brain images (size 96x512x512), 2 computerized tomography(CT) rat brain images (size $337 \times 339 \times 341$ ) and 5 original magnetic 
resonance imaging(MRI) images(size 256×256x300) obtained from Queen medical center in Nottingham (United Kingdom). Otsu thresholds used for heart vessel segmentation range between 1 to 33 . Otsu thresholds 1 to 28 were used for segmentation of human brain and carotid vessels. Hysteresis threshold used for rat brain, carotid vessels and heart vessels segmentation range between 0 and 1. WTHBHVEF Parameters such as the scale size $(n=1,3,5,7,9)$ were used during the segmentation process. other WTHBHVEF Parameters used during segmentation of heart vessels include $\alpha=0.25$, $\beta=90$, and $c=500 . \alpha=0.2, \beta=50$,and $c=500$ were used during segmentation of both brain vessel and carotid segmentation since vessels give a higher response at those scales and parameters. Bilateral parameter that were used for both heart vessel and brain vessel and carotid segmentation include: $\sigma_{r}=2$ and $\sigma_{s}=68$. where $\sigma_{s}$ is the width of spatial Gaussian. $\sigma_{r}$ is the width of range Gaussian.

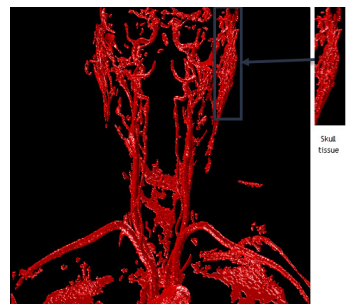

(a)

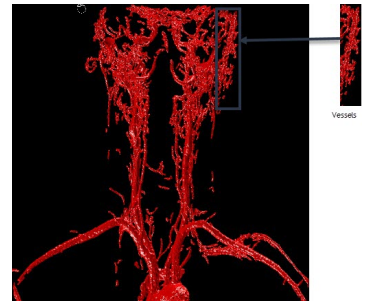

(b)
Fig. 2. Visual comparison of different segmentation techniques on ce-MRA neck and brain dataset for skull removal. (a) carotid segmentation using multithreshold Otsu method with respect to skull removal, (b) carotid segmentation using multi-threshold Otsu and hysteresis thresholding method with respect to skull removal.

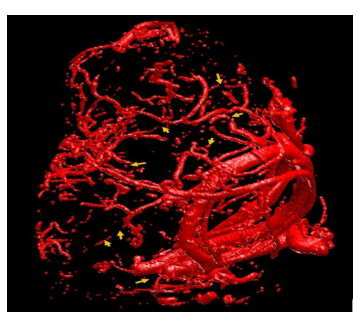

(a)

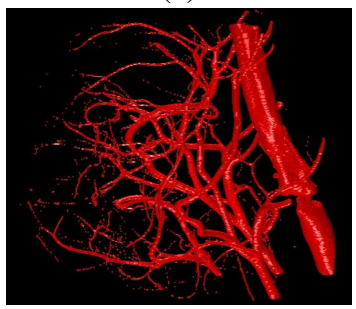

(c)

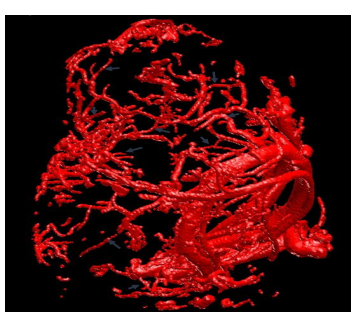

(b)

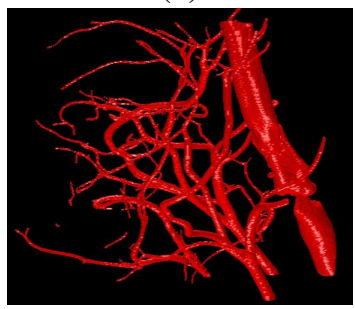

(d)
Fig. 3. Visual comparison of multi-threshold Otsu method and a combination of multi-threshold Otsu method and hysteresis threshold method for segmentation of medium to big vessels in MRI images and CT scan images. (a) multi-threshold Otsu on MRI images, (b) multi-threshold Otsu and hysteresis threshold method on MRI images, (c) multi-threshold Otsu on CT scan image, (d) multi-threshold Otsu method and hysteresis threshold method on CT scan image.

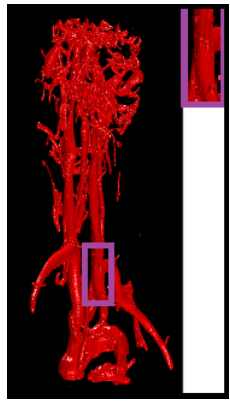

(a)

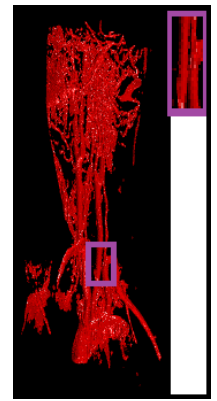

(c)

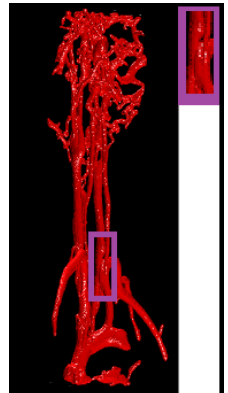

(b)

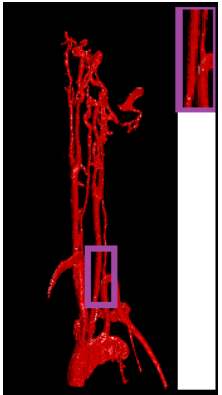

(d)
Fig. 4. Visual comparison of different segmentation techniques on ce-MRA neck and brain dataset for seperation of vessels. (a) Frangi hessian based vessel enhancement filter [71], (b) Frangi hessian based vessel enhancement filter and line filter [72], (c) multi-threshold Otsu method and WTHHVEF [41], (d) proposed method

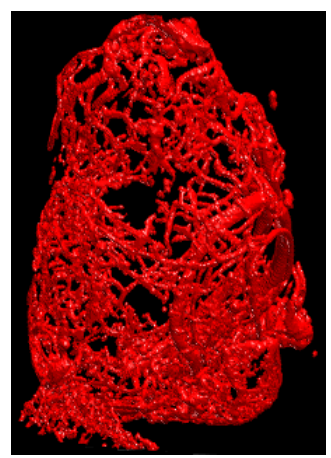

(a)

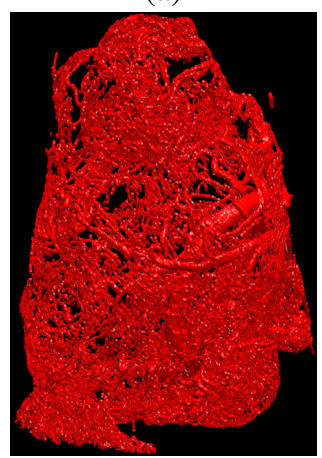

(c)

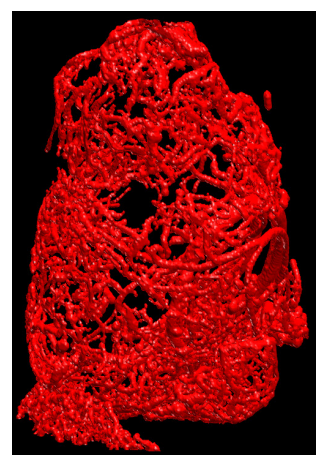

(b)

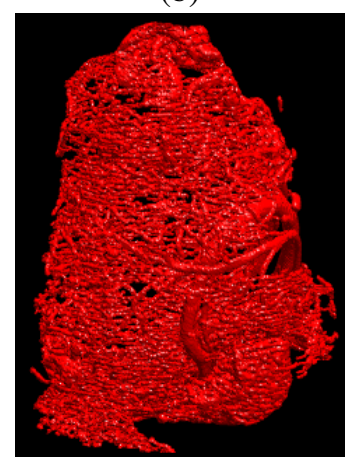

(d)
Fig. 5. Visual comparison of different segmentation techniques on MRI heart dataset. (a) Frangi hessian based vessel enhancement filter [71], (b) Frangi hessian based vessel enhancement filter and line filter [72], (c) multi-threshold Otsu method and WTHHVEF [41], (d) proposed method 


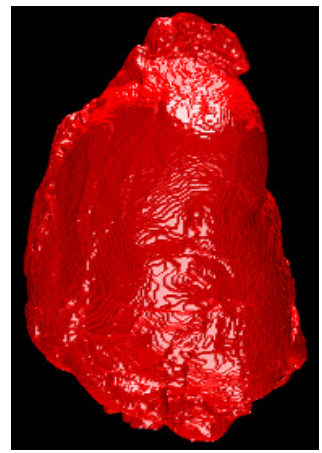

(a)

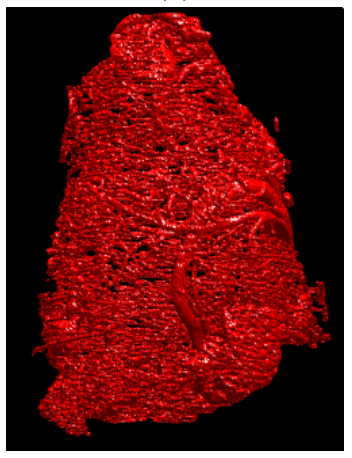

(c)

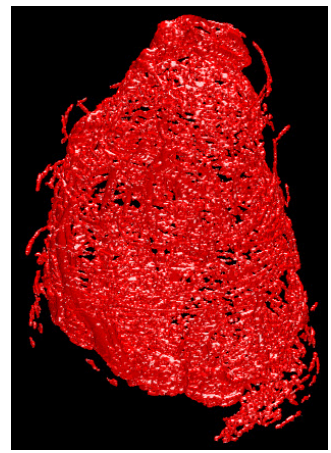

(b)

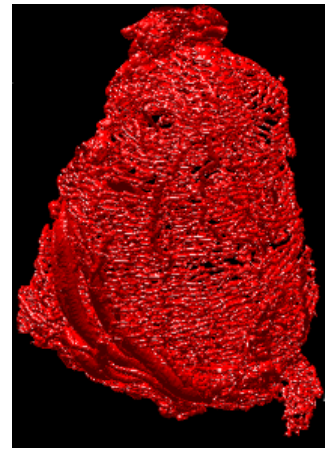

(d)
Fig. 6. Before and after vessel segmentation using the proposed method. (a) MRI heart image, (b) heart image, (b), (c) and (d) are different views of the 3D segmented image using the proposed method

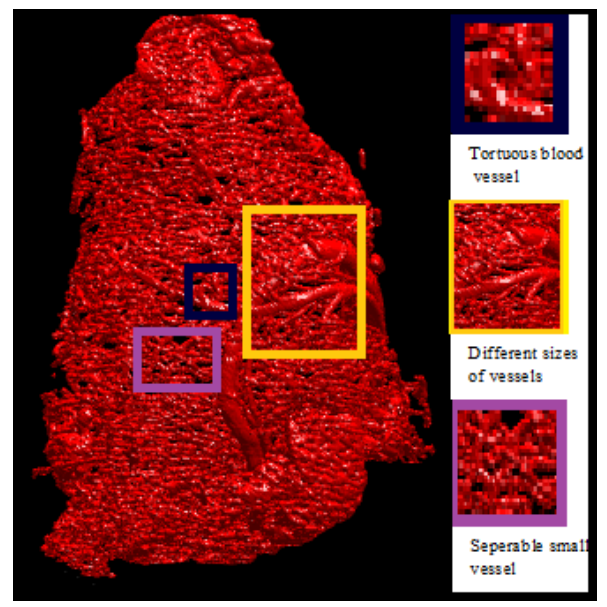

Fig. 7. After segmentation.

\section{A. Validation and Evaluation}

In medical image analysis, it is difficult to validate, quantitatively compare 3D segmentation methods as it requires ground truth to which the segmentation results can be compared. The ground truth is obtained by an expert who manually analyses and segments the medical images. Unfortunately the ground truth data is not available. In our study, a visual evaluation of segmentation results is necessary to strongly assess the contribution of this method in segmentation of MRI heart vessels and contrast enhanced-MRA Neck and brain vessels.

\section{DISCUSSION}

Based on the above results, the proposed method is capable of dealing with intensity homogeneity(Fig. 5d), More vessels are detected. Fig. 4, shows that the method can separate different sizes of vessels in both MRI and MRA medical images as compared to other methods. The skull is one of the hardest tissue to get rid of yet its one of the major crucial steps required for proper diagnosis and further analysis. The existing tools (such as FSL brain extraction tool) used by clinicians and researchers to remove skull require a lot of manual intervention. This method requires minimal human intervention (such as in adjusting threshold) to get rid of the skull and the background without interfering with vessels to ease further analysis(Fig. 2b). A combination of multthreshold Otsu method and hysteresis threshold method ensures the extraction of medium to big vessels in both MRA and MRI medical images (Fig. 3b). Yellow arrows(Fig. 3a) show vessels that are cut by multi-threshold Otsu method whilst blue arrows(Fig. 3b) show more vessels are after introducing hysteresis threshold method. MRI and MRA images are naturally detailed as compared images of other modalities (such as CT scan images). This combination (multi-threshold Otsu and Hysteresis thresholding method) does not work on the CT scan images(Fig. 3d). Less vessels are detected in CT scan images after introducing hysteresis thresholding method. Compared to other methods, this method is capable of preserving edges of different sizes of vessels(Fig. 7). In addition, the method also detects abnormalities in the vessels(Fig. 7). however, the effectiveness of the segmentation method is not quantified.

\section{CONCLUSION}

A $3 \mathrm{D}$ robust segmentation approach is presented to deal with the intensity in-homogeneity and noise in 3D medical images that suffer from intensity in-homogeneity and noise to enable extraction of vessels. A white top-hat scale space bilateral hessian based vessel enhancement filter is used to build strong edges of small to medium vessels, deal with the intensity in-homogeneity, and noise in medical images for better segmentation of small vessels. Multi-threshold Otsu is combined with hysteresis thresholding method to ensure extraction of medium to big vessels. This combination (multithreshold Otsu threshold with hysteresis threshold method) eliminates the conglutination tissue (such as the skull tissue) that obstruct segmentation process to ease segmentation of vessels. More strong edged blood vessels are detected and different sizes of vessels are separated to ease further analysis during the quantification of angiogenesis. In addition, the method is capable of removing skull tissue to ease further analysis. further more, the segmentation method can be used for diagnosis, abnormalities (such tortuous and stenosis) in the vessels are evident. The next step is to improve the method so as to work on images of other modalities (such as CT scan images) that are affected by intensity in-homogeneity. We shall quantify the effectiveness of the improved method using vessel synthetic images. We also intend to quantify angiogenesis to detect abnormal growth in vessels. 


\section{ACKNOWLEDGMENT}

Faiza Bukenya acknowledges the Islamic Development Bank (IDB) Merit scholarship scheme and University of Nottingham (United Kingdom Campus) for their generous support. We also thank Joseph Ehling, Yuchin Ding, Henryk Faas and Amir Awwad who provided the medical images for this study.

\section{REFERENCES}

[1] X. Zhang, J. Tian, K. Deng, X. Li, and F. Yang, "Graph representation of hepatic vessel based on centerline extraction and junction detection," in Medical Imaging 2012: Image Processing, vol. 8314. International Society for Optics and Photonics, 2012, p. 83143G.

[2] "MS Windows 7 heartangioplastyandstentplacement," https://www.healthline.com/health angioplasty-and-stent-placementheart, accessed on: 2018-03-3.

[3] S. P. Rajan and V. Kavitha, "Diagnosis of cardiovascular diseases using retinal images through vessel segmentation graph," Current Medical Imaging Reviews, vol. 13, no. 4, pp. 454-459, 2017.

[4] J. Zhang, Y. Cui, W. Jiang, and L. Wang, "Blood vessel segmentation of retinal images based on neural network," in International Conference on Image and Graphics. Springer, 2015, pp. 11-17.

[5] S. Khowaja, M. Unar, I. Ismaili, and P. Khuwaja, "Supervised method for blood vessel segmentation from coronary angiogram images using 7-d feature vector,' The Imaging Science Journal, vol. 64, no. 4, pp. 196-203, 2016.

[6] J. L. Even, A. F. Chen, and J. Y. Lee, "Imaging characteristics of "dynamic" versus "static" spondylolisthesis: analysis using magnetic resonance imaging and flexion/extension films," The Spine Journal, vol. 14, no. 9, pp. 1965-1969, 2014.

[7] I. Despotović, B. Goossens, and W. Philips, "Mri segmentation of the human brain: challenges, methods, and applications," Computational and mathematical methods in medicine, vol. 2015, 2015.

[8] O. Ecabert, J. Peters, M. J. Walker, T. Ivanc, C. Lorenz, J. von Berg, J. Lessick, M. Vembar, and J. Weese, "Segmentation of the heart and great vessels in ct images using a model-based adaptation framework," Medical image analysis, vol. 15, no. 6, pp. 863-876, 2011.

[9] R. Latha and S. Senthilkumar, "Robust segmentation of blood vessels from angiographic images of the human heart," in Communication and Computational Intelligence (INCOCCI), 2010 International Conference on. IEEE, 2010, pp. 174-177.

[10] P. Makowski, T. S. Sørensen, S. V. Therkildsen, A. Materka, H. Stødkilde-Jørgensen, and E. M. Pedersen, "Two-phase active contour method for semiautomatic segmentation of the heart and blood vessels from mri images for 3d visualization," Computerized Medical Imaging and Graphics, vol. 26, no. 1, pp. 9-17, 2002.

[11] A. Al-Surmi, R. Wirza, R. Mahmod, F. Khalid, and M. Z. Dimon, "A new human heart vessel identification, segmentation and $3 \mathrm{~d}$ reconstruction mechanism," Journal of cardiothoracic surgery, vol. 9, no. 1, p. 161, 2014.

[12] D. M. Greig, B. T. Porteous, and A. H. Seheult, "Exact maximum a posteriori estimation for binary images," Journal of the Royal Statistical Society. Series B (Methodological), pp. 271-279, 1989.

[13] Y. Y. Boykov and M.-P. Jolly, "Interactive graph cuts for optimal boundary \& region segmentation of objects in nd images," in Computer Vision, 2001. ICCV 2001. Proceedings. Eighth IEEE International Conference on, vol. 1. IEEE, 2001, pp. 105-112.

[14] N. Xu, N. Ahuja, and R. Bansal, "Object segmentation using graph cuts based active contours," Computer Vision and Image Understanding, vol. 107, no. 3, pp. 210-224, 2007.

[15] D. Ji, Y. Yao, Q. Yang, and X. Chen, "Mr image segmentation using graph cuts based geodesic active contours," 2016.

[16] S. M. Priyambada Subudhi, "Object segmentation using graph cuts and active contours in a pyramidal framework," pp. $10714-10714-8$, 2018. [Online]. Available: https://doi.org/10.1117/12.2299627

[17] P. Wankhede and K. Khanchandani, "Retinal blood vessel segmentation using graph cut analysis," in Industrial Instrumentation and Control (ICIC), 2015 International Conference on. IEEE, 2015, pp. 1429-1432.

[18] Z. Zhai, M. Staring, and B. C. Stoel, "Lung vessel segmentation in ct images using graph-cuts," in Medical Imaging 2016: Image Processing, vol. 9784. International Society for Optics and Photonics, 2016, p. $97842 \mathrm{~K}$.
[19] Y. Zhao, Y. Liu, X. Wu, S. P. Harding, and Y. Zheng, "Retinal vessel segmentation: an efficient graph cut approach with retinex and local phase," PloS one, vol. 10, no. 4, p. e0122332, 2015.

[20] J. Zheng, P.-R. Lu, D. Xiang, Y.-K. Dai, Z.-B. Liu, D.-J. Kuai, H. Xue, and Y.-T. Yang, "Retinal image graph-cut segmentation algorithm using multiscale hessian-enhancement-based nonlocal mean filter," Computational and mathematical methods in medicine, vol. 2013, 2013.

[21] N. Sharma and L. M. Aggarwal, "Automated medical image segmentation techniques," Journal of medical physics/Association of Medical Physicists of India, vol. 35, no. 1, p. 3, 2010.

[22] Y. Yin, M. Adel, and S. Bourennane, "Retinal vessel segmentation using a probabilistic tracking method," Pattern Recognition, vol. 45, no. 4, pp. 1235-1244, 2012.

[23] H. E. Cline, D. R. Thedens, P. Irarrazaval, C. H. Meyer, B. S. Hu, D. G. Nishimura, and S. Ludke, "3d mr coronary artery segmentation," Magnetic resonance in medicine, vol. 40, no. 5, pp. 697-702, 1998.

[24] N. Flasque, M. Desvignes, J.-M. Constans, and M. Revenu, "Acquisition, segmentation and tracking of the cerebral vascular tree on $3 \mathrm{~d}$ magnetic resonance angiography images," Medical Image Analysis, vol. 5, no. 3, pp. 173-183, 2001.

[25] H. J. Noordmans and A. W. Smeulders, "High accuracy tracking of 2d/3d curved line-structures by consecutive cross-section matching1," Pattern Recognition Letters, vol. 19, no. 1, pp. 97-111, 1998.

[26] M. Cree, D. Cornforth, and H. Jelinek, "Vessel segmentation and tracking using a two-dimensional model."

[27] C. Ortiz de Solorzano, R. Malladi, S. Lelievre, and S. Lockett, "Segmentation of nuclei and cells using membrane related protein markers," journal of Microscopy, vol. 201, no. 3, pp. 404-415, 2001.

[28] G. Xiong, X. Zhou, L. Ji, P. Bradley, N. Perrimon, and S. Wong, "Segmentation of drosophila rnai fluorescence images using level sets," in Image Processing, 2006 IEEE International Conference on. IEEE, 2006, pp. 73-76.

[29] S. Lu, H. Huang, P. Liang, G. Chen, and L. Xiao, "Hepatic vessel segmentation using variational level set combined with non-local robust statistics," Magnetic Resonance Imaging, vol. 36, p. 180(7), 2017-02-01.

[30] B. Dizdaroğlu, E. Ataer-Cansizoglu, J. Kalpathy-Cramer, K. Keck, M. F. Chiang, and D. Erdogmus, "Structure-based level set method for automatic retinal vasculature segmentation," EURASIP Journal on Image and Video Processing, vol. 2014, no. 1, p. 39, 2014.

[31] P. Thevenaz and M. Unser, "Snakuscules," IEEE Transactions on image processing, vol. 17, no. 4, pp. 585-593, 2008

[32] K. Krissian, G. Malandain, N. Ayache, R. Vaillant, and Y. Trousset, "Model-based detection of tubular structures in 3d images," Computer vision and image understanding, vol. 80, no. 2, pp. 130-171, 2000.

[33] D.-C. Cheng, C. Billich, S.-H. Liu, H. Brunner, Y.-C. Qiu, Y.-L. Shen, H. J. Brambs, A. Schmidt-Trucksäss, and U. H. Schütz, "Automatic detection of the carotid artery boundary on cross-sectional mr image sequences using a circle model guided dynamic programming," Biomedical engineering online, vol. 10, no. 1, p. 26, 2011.

[34] I. Bitter, R. Van Uitert, I. Wolf, E. Tzatha, A. M. Gharib, R. Summers, H.-P. Meinzer, and R. Pettigrew, "Virtual contrast for coronary vessels based on level set generated subvoxel accurate centerlines," International journal of biomedical imaging, vol. 2006, 2006.

[35] S.-T. Chen, T.-D. Wang, W.-J. Lee, T.-W. Huang, P.-K. Hung, C.-Y. Wei, C.-M. Chen, and W.-M. Kung, "Coronary arteries segmentation based on the $3 \mathrm{~d}$ discrete wavelet transform and $3 \mathrm{~d}$ neutrosophic transform," BioMed research international, vol. 2015, 2015.

[36] R. Manniesing, M. A. Viergever, and W. J. Niessen, "Vessel axis tracking using topology constrained surface evolution," IEEE Transactions on Medical Imaging, vol. 26, no. 3, pp. 309-316, 2007.

[37] D. D. Xin Hu, Yuanzhi Cheng and D. Chu, "Axis-guided vessel segmentation using a self-constructing cascade-adaboost-svm classifier," BioMed Research International, vol. 2018, no. 3636180, p. 12, 2018.

[38] S. Wörz and K. Rohr, "3d adaptive model-based segmentation of human vessels," in Medical Imaging 2007: Physiology, Function, and Structure from Medical Images, vol. 6511. International Society for Optics and Photonics, 2007, p. 65110Q.

[39] B. Mathew, A. Schmitz, S. Muñoz-Descalzo, N. Ansari, F. Pampaloni, E. H. Stelzer, and S. C. Fischer, "Robust and automated threedimensional segmentation of densely packed cell nuclei in different biological specimens with lines-of-sight decomposition," BMC bioinformatics, vol. 16, no. 1, p. 187, 2015.

[40] J. D. Ross, D. K. Cullen, J. P. Harris, M. C. LaPlaca, and S. P. DeWeerth, "A three-dimensional image processing program for accurate, rapid, and 
semi-automated segmentation of neuronal somata with dense neurite outgrowth," Frontiers in neuroanatomy, vol. 9, p. 87, 2015.

[41] F. Bukenya, J. Ehling, A. Kalema, I. Eyoh, J. Robert, and L. Bai, " $3 \mathrm{~d}$ segmentation of the whole heart vasculature using improved multithreshold otsu and white top-hat scale space hessian based vessel filter,' in 2016 IEEE Symposium Series on Computational Intelligence (SSCI), Dec 2016, pp. 1-7.

[42] G. A. Almira, T. Harsono, R. Sigit, I. G. N. T. B. Bimantara, and J. S. M. Saputra, "Performance analysis of gaussian and bilateral filter in case of determination the fetal length," Knowledge Creation and Intelligent Computing (KCIC), 2016.

[43] C. Ye, D. Tao, M. Song, D. W. Jacobs, and M. Wu, "Sparse norm filtering," arXiv preprint arXiv:1305.3971, 2013.

[44]

[45] K. N. Chaudhury and S. D. Dabhade, "Fast and provably accurate bilateral filtering," IEEE Transactions on Image Processing, vol. 25 no. 6, pp. 2519-2528, 2016.

[46] D. L. Buckeridge, H. Burkom, M. Campbell, W. R. Hogan, A. W. Moore et al., "Algorithms for rapid outbreak detection: a research synthesis," Journal of biomedical informatics, vol. 38, no. 2, pp. 99-113, 2005.

[47] C. Yu, C. Dian-ren, L. Yang, and C. Lei, "Otsu's thresholding method based on gray level-gradient two-dimensional histogram," in Informatics in Control, Automation and Robotics (CAR), 2010 2nd International Asia Conference on, vol. 3. IEEE, 2010, pp. 282-285.

[48] L. Wang, H. Duan, and J. Wang, "A fast algorithm for three-dimensional otsu's thresholding method," in IT in Medicine and Education, 2008. ITME 2008. IEEE International Symposium on. IEEE, 2008, pp. 136140.

[49] F. Bukenya, L. Bai, and A. Kiweewa, "A review of blood vessel segmentation techniques," in 2018 1st International Conference on Computer Applications Information Security (ICCAIS), April 2018, pp. $1-10$.

[50] X. Yuan, J.-F. Martínez, M. Eckert, and L. López-Santidrián, "An improved otsu threshold segmentation method for underwater simultaneous localization and mapping-based navigation," Sensors, vol. 16, no. 7, p. 1148, 2016.

[51] P. Sthitpattanapongsa and T. Srinark, "An equivalent 3d otsu's thresholding method," in Pacific-Rim Symposium on Image and Video Technology. Springer, 2011, pp. 358-369.

[52] D. Selvaraj and R. Dhanasekaran, "Mri brain image segmentation techniques-a review."

[53] A. S. Abutaleb, "Automatic thresholding of gray-level pictures using two-dimensional entropy," Computer vision, graphics, and image processing, vol. 47, no. 1, pp. 22-32, 1989.

[54] X. He, Z. Pan, Q. Dong, and G. Wang, "Veins segmentation and threedimensional reconstruction from liver ct images using multilevel otsu method," in Image and Graphics (ICIG), 2013 Seventh International Conference on. IEEE, 2013, pp. 248-251.

[55] K. D. Toennies, Guide to medical image analysis. Springer.

[56] Z. Qu and L. Zhang, "Research on image segmentation based on the improved otsu algorithm," in Intelligent Human-Machine Systems and Cybernetics (IHMSC), 2010 2nd International Conference on, vol. 2. IEEE, 2010, pp. 228-231.

[57] S. Samantaa, N. Dey, P. Das, S. Acharjee, and S. S. Chaudhuri, "Multilevel threshold based gray scale image segmentation using cuckoo search," arXiv preprint arXiv:1307.0277, 2013.

[58] Z. Chen and S. Molloi, "Automatic 3d vascular tree construction in ct angiography," Computerized Medical Imaging and Graphics, vol. 27, no. 6, pp. 469-479, 2003.

[59] S. D. Kapade, "Swarm intelligence based graph partitioning for image segmentation," 2015.

[60] L. Xie, M. A. Sparks, W. Li, Y. Qi, C. Liu, T. M. Coffman, and G. A. Johnson, "Quantitative susceptibility mapping of kidney inflammation and fibrosis in type 1 angiotensin receptor-deficient mice," $N M R$ in biomedicine, vol. 26, no. 12, pp. 1853-1863, 2013.

[61] R. Medina-Carnicer, A. Carmona-Poyato, R. Muñoz-Salinas, and F. J. Madrid-Cuevas, "Determining hysteresis thresholds for edge detection by combining the advantages and disadvantages of thresholding methods," IEEE transactions on image processing, vol. 19, no. 1, pp. 165173,2010

[62] O. Pereira, E. Torre, Y. Garcés, and R. Rodríguez, "Edge detection based on kernel density estimation," arXiv preprint arXiv:1411.1297, 2014.
[63] S. B. Emrani, Zahra and H. Rabbani, "A new parallel approach for accelerating the gpu-based execution of edge detection algorithms,' Journal of Medical Signals and Sensors, vol. 7, no. 1, p. 33-42, 2017.

[64] C.-H. Yeh, C.-Y. Lin, K. Muchtar, H.-E. Lai, and M.-T. Sun, "Threepronged compensation and hysteresis thresholding for moving object detection in real-time video surveillance," IEEE Transactions on Industrial Electronics, vol. 64, no. 6, pp. 4945-4955, 2017.

[65] "MS Windows 7 why is hysteresis thresholding important for edge detection? is it absolutely necessary?" https://www.quora.com/Whyis-hysteresis-thresholding-important-for-edge-detection-Is-it-absolutelynecessary , accessed on: 2018-04-14.

[66] N. M. Zaitoun and M. J. Aqel, "Survey on image segmentation techniques," Procedia Computer Science, vol. 65, pp. 797-806, 2015.

[67] K. BahadarKhan, A. A. Khaliq, and M. Shahid, "A morphological hessian based approach for retinal blood vessels segmentation and denoising using region based otsu thresholding," PloS one, vol. 11, no. 7, p. e0158996, 2016.

[68] "Segmentation of retinal blood vessels by top-hat multi-scale detection for optic disc removal," 2015.

[69] A. C. Jalba, M. H. Wilkinson, and J. B. Roerdink, "Morphological hattransform scale spaces and their use in pattern classification," Pattern Recognition, vol. 37, no. 5, pp. 901-915, 2004.

[70] C. Tomasi and R. Manduchi, "Bilateral filtering for gray and color images," in Computer Vision, 1998. Sixth International Conference on. IEEE, 1998, pp. 839-846.

[71] A. F. Frangi, W. J. Niessen, K. L. Vincken, and M. A. Viergever, "Multiscale vessel enhancement filtering," in International Conference on Medical Image Computing and Computer-Assisted Intervention. Springer, 1998, pp. 130-137.

[72] Y. Ding, W. Ward, T. Wästerlid, P. Gowland, A. Peters, J. Yang, S. Nakagawa, and L. Bai, "Three-dimensional vessel segmentation using a novel combinatory filter framework," Physics in Medicine \& Biology, vol. 59, no. 22, p. 7013, 2014. 